\title{
A $B_{1}$-Insensitive High Resolution 2D $T_{1}$ Mapping Pulse Sequence for dGEMRIC of the HIP at 3 Tesla
}

\author{
Riccardo Lattanzi ${ }^{1,2,{ }^{*}, \text { Christian Glaser }}{ }^{1,2}$, Artem V. Mikheev ${ }^{2}$, Catherine Petchprapa ${ }^{2}$, David \\ J. Mossa ${ }^{2}$, Soterios Gyftopoulos ${ }^{2}$, Henry Rusinek ${ }^{2}$, Michael Recht ${ }^{2}$, and Daniel Kim ${ }^{1,2}$ \\ ${ }^{1}$ Department of Radiology, Center for Biomedical Imaging, New York University Langone Medical \\ Center, New York, New York, USA \\ 2Department of Radiology, New York University Langone Medical Center, New York, New York, \\ USA
}

\section{Abstract}

Early detection of cartilage degeneration in the hip may help prevent onset and progression of osteoarthritis in young patients with femoroacetabular impingement. Delayed gadoliniumenhanced MRI of cartilage is sensitive to cartilage glycosaminoglycan loss and could serve as a diagnostic tool for early cartilage degeneration. We propose a new high resolution 2D $T_{1}$ mapping saturation-recovery pulse sequence with fast spin echo readout for delayed gadolinium-enhanced magnetic resonance imaging of cartilage of the hip at $3 \mathrm{~T}$. The proposed sequence was validated in a phantom and in 10 hips, using radial imaging planes, against a rigorous multipoint saturationrecovery pulse sequence with fast spin echo readout. $T_{1}$ measurements by the two pulse sequences were strongly correlated $\left(R^{2}>0.95\right)$ and in excellent agreement (mean difference $=-8.7 \mathrm{~ms}$; upper and lower $95 \%$ limits of agreement $=64.5$ and $-81.9 \mathrm{~ms}$, respectively). $T_{1}$ measurements were insensitive to $B_{1+}$ variation as large as $20 \%$, making the proposed $T_{1}$ mapping technique suitable for $3 \mathrm{~T}$.

\section{Keywords}

femoroacetabular impingement; MRI; hip, cartilage; dGEMRIC; radial MRI; $T_{1}$ mapping; $B_{1+}$ inhomogeneity

\begin{abstract}
In recent years, it has been increasingly recognized that femoroacetabular impingement (FAI), a condition in which structural abnormalities of the femoral head-neck junction and/or acetabulum cause mechanical blockage in the terminal range of hip motion, can lead to osteoarthritis of the hip $(1,2)$. In FAI, the abnormal contact between the acetabular rim and femoral neck causes chondral and labral damage, which can progress over time and result in osteoarthritis of the hip joint if the underlying cause of impingement is not addressed surgically (3).
\end{abstract}

\footnotetext{
*Correspondence to: Riccardo Lattanzi, Ph.D., Center for Biomedical, Imaging, New York University School of Medicine, 660 First Avenue, Fourth Floor, New York, New York 10016. riccardo.lattanzi@ nyumc.org.
} 
MR Imaging has emerged as the preferred diagnostic modality for suspected FAI due to its multiplanar image acquisition capability and its high soft tissue contrast. The acetabular cartilage's and labrum's position and orientation within the pelvis make MR imaging of these structures in three orthogonal planes susceptible to partial volume effects. One approach to minimize partial volume averaging is to image the acetabular rim and cartilage in at set of rotating radial planes (4). Imaging in rotating radial planes exploits the geometry of the hip joint and allows orthogonal display of the whole acetabular rim around its circumference. This imaging technique has been shown to be useful in identifying obliquely oriented tears in the anterosuperior and posterosuperior sections of the labrum (4).

Corrective surgical procedures aimed at removing the bony abnormalities of FAI and treating the associated labral and cartilage abnormalities are less likely to be successful in patients presenting with extensive articular cartilage injuries (5), for whom the only viable treatment is arthroplasty. Therefore, it is imperative to detect cartilage damage in its early stages. Cartilage that appears morphologically normal in routine MRI may already be irreversibly compromised in early osteoarthritis. MR-based biochemical imaging techniques, such as delayed gadolinium-enhanced MRI of cartilage (dGEMRIC) $(6,7)$, have been proposed as an early diagnostic tool for the evaluation of chondral lesions. In dGEMRIC, negatively charged contrast agent $\left(\mathrm{Gd}-\mathrm{DTPA}^{2-}\right)$ is administered prior to an exercise protocol, to exploit the different Gd-DTPA kinetics between the healthy and compromised cartilage, and imaging is performed to measure delayed contrast enhancement of compromised cartilage, which reflects the local concentration of glycosaminoglycans in an inverse relationship $(6,7)$. The areas with depleted glycosaminoglycans will have higher concentrations of Gd-DTPA ${ }^{2-}$, which will be reflected in the measured $T_{1}$. Therefore, dGEMRIC provides an indirect visualization of glycosaminoglycans loss, which is an early sign of cartilage degeneration (8).

In recent years, a fast 2-angle $T_{1}$ mapping (F2T1) pulse sequence based on threedimensional (3D) gradient echo readout has been introduced (9) and validated (10) for dGEMRIC in the hip. The F2T1 pulse sequence is more time-efficient than two-dimensional (2D) multipoint inversion recovery and saturation-recovery (SR) pulse sequences, which are problematic for clinical use due to their long acquisition times $(8,11)$. The F2T1 sequence has been proposed to acquire dGEMRIC datasets covering the entire hip joint with isotropic spatial resolution, which can then be reformatted during postprocessing in rotating radial planes of the hip joint $(12,13)$. These studies showed that dGEMRIC images reformatted during postprocessing in rotating radial planes depict cartilage damage in the anteriorsuperior region of the acetabulum (12), where cartilage injury typically occurs in FAI patients. These previously reported 3D dGEM-RIC results were obtained at $1.5 \mathrm{~T}$ with approximately $0.80 \mathrm{~mm} \times 0.80 \mathrm{~mm} \times 0.80 \mathrm{~mm}$ isotropic spatial resolution and acquisition times in the order of 9-10 min $(12,13)$ or more, depending on the number of partitions needed to sample the whole 3D volume without aliasing artifacts. Given the small dimensions of hip acetabular cartilage (14), it may be necessary to further increase the spatial resolution and reduce the scan time to minimize the loss in spatial resolution due to patient motion. One approach to increase the spatial resolution and/or reduce the scan time is to perform 3D dGEMRIC at $3 \mathrm{~T}$ and trade increased signal-to-noise ratio (SNR) for higher resolution and/or faster imaging (e.g., higher acceleration), respectively, at the expense of 
reduced accuracy due to increased $B_{1+}$ variation within the hip at $3 \mathrm{~T}$. The loss in accuracy can be partially compensated with a corresponding $B_{1+}$ mapping method $(15,16)$, where the resulting flip angle maps can be used to correct the $T_{1}$ map.

We propose to exploit increased SNR afforded by $3 \mathrm{~T}$ imaging by performing high spatial resolution $2 \mathrm{D} T_{1}$ mapping in radial imaging planes to take advantage of the geometry of the hip joint $(4,12)$. Therefore, the aim of this study was to develop a new $B_{1}$-insensitive $2 \mathrm{D} T_{1}$ mapping pulse sequence with high in-plane resolution for dGEMRIC in radial planes of the hip. Our approach was to image the hip using a fast spin echo (FSE) pulse sequence at $3 \mathrm{~T}$ to achieve high spatial resolution with adequate SNR and employ a $B_{1}$-insensitive saturation pulse to perform uniform $T_{1}$ weighting. The scan time of the proposed pulse sequence is 1 min and $20 \mathrm{~s}$ per 2D slice. Compared with the previously reported 3D dGEM-RIC pulse sequence, the proposed pulse sequence is relatively less sensitive to patient motion. We validated the proposed pulse sequence against a rigorous multipoint SR pulse sequence at 3 $\mathrm{T}$, by comparing measured $T_{1}$ in a phantom and in the hip cartilage of FAI patients. We also compared the accuracy and SNR efficiency of the propose pulse sequence against the 3D F2T1 pulse sequence in phantom experiments.

\section{MATERIALS AND METHODS}

\section{Pulse Sequence}

We modified a FSE pulse sequence to perform two image acquisitions with two different $T_{1}$ weightings. The first FSE image acquisition was acquired after applying a saturation pulse with a SR time delay (TD) on the order of $T_{1}$, to achieve a good balance between $T_{1}$ sensitivity and SNR. Based on previous dGEMRIC studies at $1.5 \mathrm{~T}$ (13) and $3 \mathrm{~T}$ (17), we anticipated $T_{1}$ of normal cartilage at $3 \mathrm{~T}$ to be on the order of $700-800 \mathrm{~ms}$. As such, we used $\mathrm{TD}=700 \mathrm{~ms}$ to achieve a good balance between $T_{1}$ sensitivity and SNR for the SR acquisition (18). In the proposed $\mathrm{SR}$ acquisition with $\mathrm{TD}=700 \mathrm{~ms}$, tissues with relatively short $T_{1}$ values ( $<350 \mathrm{~ms}$ ), for which the recovery of the longitudinal magnetization is nearly complete, and tissues with long $T_{1}$ values ( $>2100 \mathrm{~ms}$ ), for which the recovery of the magnetization is minimal, are especially susceptible to random error. The second FSE image [i.e., proton density (PD)] acquisition was performed with TR on the order of $5 T_{1} \mathrm{~s}$ and without the saturation pulse. $T_{1}$ was calculated pixel-wise by dividing the SR image, $I_{\mathrm{SR}}$, by the PD image, $I_{\mathrm{PD}}$, to correct for the unknown equilibrium magnetization $\left(M_{0}\right)$, and then solving the ideal SR experiment described by the Bloch equation governing $T_{1}$ relaxation:

$$
\frac{I_{\mathrm{SR}}=M_{0}\left(1-e^{-\mathrm{TD} / T_{1}}\right)}{I_{\mathrm{PD}}=M_{0}}=\left(1-e^{-\mathrm{TD} / T_{1}}\right),
$$

$$
T_{1}=\frac{-T D}{\log \left(1-\frac{I_{S R}}{I_{P D}}\right)}
$$


The modified FSE pulse sequence was implemented on a whole-body 3 T MRI scanner (Verio, Siemens Healthcare, Erlangen, Germany) equipped with a gradient system capable of achieving a maximum gradient strength of $45 \mathrm{mT} / \mathrm{m}$ and a slew rate of $200 \mathrm{~T} / \mathrm{m} / \mathrm{s}$. The radiofrequency (RF) excitation was performed using the transmit body coil, and a 32-element "cardiac" coil array (Invivo, Orlando, FL) was employed for signal reception. The relevant imaging parameters are: field of view $=190 \mathrm{~mm} \times 190 \mathrm{~mm}$, acquisition matrix $=320 \times 320$, in-plane resolution $=0.6 \mathrm{~mm} \times 0.6 \mathrm{~mm}$, slice thickness $=5 \mathrm{~mm}$, turbo factor $=13$, FSE readout duration was $143 \mathrm{~ms}, \mathrm{TE}=10 \mathrm{~ms}$, refocusing flip angle was $180^{\circ}$, generalized autocalibrating partially parallel acquisitions (19) with an acceleration factor $=1.8$ and receiver bandwidth $=161 \mathrm{~Hz} /$ pixel . A fat suppression pulse was used to avoid chemical shift artifacts at the bone-cartilage interface. TR (including the saturation pulse, recovery time, and FSE readout duration) was $850 \mathrm{~ms}$ and $4000 \mathrm{~ms}$ for SR and PD acquisitions, respectively. Total scan time for both SR and PD acquisitions was 1 min $20 \mathrm{~s}$ per slice.

We used a $B_{1}$-insensitive saturation pulse (20) to achieve uniform $T_{1}$ weighting within the hip at $3 \mathrm{~T}$. The hybrid adiabatic-rectangular pulse train is comprised of three nonselective RF pulses: nonselective rectangular $140^{\circ}$ pulse, nonselective rectangular $90^{\circ}$ pulse, and nonselective adiabatic half-passage pulse. The crusher gradients inserted between RF pulses are cycled to eliminate stimulated echoes. Spoiler gradients are applied before the first RF pulse and after the third RF pulse to dephase the transverse magnetization.

To validate the $T_{1}$ measurements calculated with Eq. 2, we acquired four additional SR images, with $\mathrm{TD}=350,1050,1750$, and $2450 \mathrm{~ms}$ (Fig. 1). Total scan time for the four additional SR images was $1 \mathrm{~min} 40 \mathrm{~s}$ per slice. These additional SR images were combined with the aforementioned SR image with TD $=700 \mathrm{~ms}$ and the PD image, to perform a twoparameter $\left(M_{0}, T_{1}\right)$ nonlinear fit of Eq. 1 . All six images were acquired in series to minimize image registration errors. Total scan time to acquire all six images was 3 min per slice.

We compared the proposed 2D FSE pulse sequence against the 3D F2T1 pulse sequence in two phantom experiments. For the first phantom experiment designed to compare their sensitivity to $B_{1+}$ variations, 2D $T_{1}$ mapping pulse sequence was performed with the aforementioned protocol, and 3D F2T1 imaging was performed with the following parameters: spatial resolution $=0.8 \mathrm{~mm} \times 0.8 \mathrm{~mm} \times 0.8 \mathrm{~mm}$, flip angles $=5$ and $30^{\circ}, \mathrm{TE} / \mathrm{TR}$ $=3.5 / 20 \mathrm{~ms}$, receiver bandwidth $=130 \mathrm{~Hz} /$ pixel, 144 partitions, $22 \%$ partition over sampling, $41 \%$ partition over sampling, generalized autocalibrating partially parallel acquisitions acceleration factor $=1.8$, partial Fourier factor $6 / 8$ in the phase-encoding direction, and scan time $=13 \mathrm{~min} 16 \mathrm{~s}$. Prior to the 3D F2T1 sequence, a $B_{1+}$ mapping prescan, based on a stimulated echo pulse sequence $(15,16)$, was performed to correct the $T_{1}$ maps calculated from the 3D F2T1 images. The $T_{1}$ maps with $B_{1+}$ correction were computed using the Siemens inline reconstruction algorithm on our $3 \mathrm{~T}$ scanner equipped with VB17 software platform. For the second phantom experiment, designed to compare their SNR efficiencies, both our proposed 2D $T_{1}$ mapping and 3D F2T1 mapping methods were performed with full $k$-space encoding (i.e., no generalized autocalibrating partially parallel acquisitions acceleration and no partial Fourier imaging), where the scan time was 2 min and $15 \mathrm{~s}$ and $31 \mathrm{~min}$ and $48 \mathrm{~s}$, respectively, to calculate the SNR as the ratio of the mean signal and standard deviation of background noise (21). 


\section{Phantom Imaging}

We imaged a spherical mineral oil phantom with a known $T_{1}(\sim 550 \mathrm{~ms})$ in the coronal plane to determine the sensitivity of the saturation pulse to clinically relevant $B_{1+}$ variations within the hip at $3 \mathrm{~T}$. To avoid signal saturation of the oil phantom, the phantom experiment was performed without the fat suppression pulse. Image acquisition was repeated with $B_{1+}$ scale of the saturation pulse manually adjusted from 0.8 to 1.2 (0.1 steps) of its nominally calibrated $B_{1+}$ value. Nominal $B_{1+}$ was determined using the automated RF transmit calibration procedure. The upper limit of $20 \% B_{1+}$ variation was based on our preliminary experience with hip imaging at $3 \mathrm{~T}$.

In the second experiment, the phantom was comprised of approximately $9 \%$ glycerol in distilled water to emulate relaxation times of hip cartilage (measured $T_{1}=730 \mathrm{~ms}$; measured $\mathrm{T}_{2}=37 \mathrm{~ms}$ ). For the 3D data, SNR was measured only in a 2D plane that corresponds to the 2D FSE plane. To account for the difference in voxel sizes, we normalized the SNR by the voxel size. The normalized SNR efficiency was then calculated as the normalized SNR divided by the square root of the scan time.

\section{Hip Imaging}

Patients with hip pain and positive physical examination for FAI were imaged after a double dose $(0.2 \mathrm{mmol} / \mathrm{kg})$ intravenous injection of Gd-DTPA ${ }^{2-}$ (Magnevist ${ }^{\circledR}$, Bayer Healthcare) and 15 min walking on a treadmill at controlled speed (9). The dGEMRIC pulse sequence was applied after the clinical protocol, $45 \mathrm{~min}$ after administration of Gd-DTPA. Ten hips ( 6 left, 4 right) were scanned in nine consecutive patients (mean age $=36 \pm 10$ years). All images were acquired in a radial plane that included the anterior-superior region of the acetabulum. Human imaging was performed in accordance with protocols approved by the Human Investigation Committee at our institution; all subjects provided written informed consent.

\section{Image Analysis}

Image processing was performed using an in-house developed software. For each hip, the six images acquired at different time points (see Fig. 1) were spatially registered to the PD image to compensate for motion. In particular, we used affine transformation to register only a user-defined region of interest (ROI) comprising the entire hip joint.

After deidentification and randomization of the patient data, two observers (RL and DK) manually segmented a ROI over the weight-bearing portion of the hip articular cartilage (10), extending from the lateral bony edge, not including the labrum, to the edge of the acetabular fossa. For each ROI, the software calculated an analytically solved $T_{1}$ map based on the formula in Eq. 2 (using TD $=700 \mathrm{~ms}$ and PD). As a reference measurement, the software also calculated a two-parameter six-point fitted $T_{1}$ map based on Eq. 1, using all six images and a global optimization algorithm (22). Observer 1 (RL) repeated the image analysis after 14 days from the first analysis to assess intraobserver variability. Interobserver variability was assessed between observer 1 and observer 2 (DK), comparing the average $T_{1}$ value in the cartilage ROI for each hip. The two independent observers were blinded to patient identity and each other. 


\section{Statistical Analysis}

For each ROI, the difference between the analytic $T_{1}$ and the six-point fit $T_{1}$ was calculated pixel-wise to display the spatial distribution of error for each analysis session. We performed the Pearson correlation and Bland-Altman (23) analyses using the mean $T_{1}$ value in each ROI.

\section{Noise Analysis}

To estimate the $T_{1}$ error, we performed a theoretical analysis using Eq. 1 for reference $T_{1}$ mapping (i.e., 6-point SR experiment) and Eq. 2 for analytic $T_{1}$ mapping, as a function of true $T_{1}$ ranging from 600 to $1200 \mathrm{~ms}$ (5 ms steps). The lower (normal-200 ms) and upper (normal $+400 \mathrm{~ms}$ ) limits of the $T_{1}$ range were based on assuming normal cartilage $T_{1}$ equal to $800 \mathrm{~ms}$ (see results). To estimate clinically relevant white Gaussian noise, in a 27 -year-old male volunteer, we acquired two PD image acquisitions in radial planes of the hip with full $k$-space encoding and TR $=10 \mathrm{~s}\left(>5 T_{1}\right)$. In addition, we acquired a noise map using the same pulse sequence without RF excitation. The hip articular cartilage was segmented manually, and the SNR was calculated as the ratio of the mean cartilage signal and standard deviation of noise derived from the noise map. The average of two PD SNR measurements was 127.5. Given that our proposed PD acquisition performs generalized autocalibrating partially parallel acquisitions acceleration 1.8, we anticipate a PD SNR of 95. Assuming $M_{0}$ $=\mathrm{PD}$, clinically relevant white Gaussian noise was estimated as $0.0105 M_{0}\left(=M_{0} / 95\right)$. The theoretical noise analysis was repeatedly performed 100 times using a numerical phantom with 100 pixels to mimic the typical number of pixels in the segmented hip cartilage, where identical amount of white noise was added to the numerical PD and SR images. The influence of white noise on $T_{1}$ accuracy was estimated by performing linear regression analysis on the calculated and true $T_{1}$ values and calculating root-mean-square-error. Reported linear regression statistics and root-mean-square-error values represent the mean \pm standard deviation over 100 measurements.

\section{RESULTS}

Figure 2 shows analytic and six-point $T_{1}$ maps of the phantom, as well as the percentage difference map. $T_{1}$ in the phantom was $562 \pm 21 \mathrm{~ms}$ with the analytic method and $561 \pm 15$ ms with the six-point fit method, and RMS of percent difference was $2.8 \%$, suggesting that they are quantitatively equivalent. $T_{1}$ measurements with the analytic method were 567, 565, 561,561 , and $563 \mathrm{~ms}$ for $B_{1+}$ scales $0.8,0.9,1.0,1.1$, and 1.2, respectively. Consistent with the previous work in the heart at $3 \mathrm{~T}(20)$, the phantom $T_{1}$ values were similar throughout (less than $1 \%$ difference with respect to the average value), suggesting that the saturation pulse is insensitive to $B_{1+}$ variation as large as $20 \%$. In contrast, $T_{1}$ measurements using the 3D F2T1 pulse sequence with $B_{1+}$ correction were 559, 574, 585, 612, and $630 \mathrm{~ms}$ for $B_{1+}$ scales $0.8,0.9,1.0,1.1$, and 1.2 , respectively, suggesting that even with $B_{1+}$ correction the 3D F2T1 pulse sequence was sensitive to clinically relevant $B_{1+}$ variation (Fig. 3).

For the glycerol phantom experiment, the normalized SNR efficiency was 10.3 and 4.3 for the 2D FSE and 3D F2T1, respectively. The higher SNR efficiency of 2D FSE over 3D F2T1 
is largely due to the difference in flip angles ( $90-180^{\circ}$ vs. $5-30^{\circ}$; 2D FSE vs. 3D F2T1, respectively).

Figure 4 shows, for one representative case, the six radial images acquired with different TDs. This image series exhibits consistently good image quality. For pixels within the ROIs, global optimization (22) using the six available values allowed an accurate fitting of the SR curve shown in Fig. 1 to calculate $T_{1}$. Analytic and six-point fit $T_{1}$ maps are shown for one hip in Fig. 5, together with a map and a histogram of the percent difference between the two. The range of the color bars were chosen to span the distribution of values in the ROIs. In this particular hip, the pixel-wise percent difference between analytic and six-point fit $T_{1}$ ranged from -6.4 to $6.8 \%$, and the RMS of percent difference was $3.2 \%$.

The mean $T_{1}$ over 10 hips was $823 \pm 189 \mathrm{~ms}, 808 \pm 183 \mathrm{~ms}$, and $797 \pm 132 \mathrm{~ms}$, for the two sessions of observer 1 and the single session of observer 2, respectively. The fact that mean $T_{1}$ of cartilage was on the order of $800 \mathrm{~ms}$ confirms our choice in TD of $700 \mathrm{~ms}$. The top row of Figure 6 shows the correlation between analytic and six-point fit $T_{1}$ for the 10 hips, whereas the bottom row shows Bland-Altman plots that illustrate the agreement between the two $T_{1}$ measurements. The Person correlation coefficient of determination $R^{2}$ was larger than 0.95 in all cases $(P<0.001)$, suggesting that the two measurements were strongly correlated. According to the Bland-Altman analysis, analytic and six-point fit $T_{1}$ values were in good agreement (mean difference $=-8.7 \mathrm{~ms}$, i.e., $\sim 1 \%$; upper and lower $95 \%$ limits of agreement $=64.5$ and $-81.9 \mathrm{~ms}$, respectively). Pearson and Bland-Altman statistics for observer 1, analysis 2, and observer 2 are shown in Table 1. As summarized in Table 1, the intra/interobserver variability in $T_{1}$ calculated from the same SR data with the analytic method was $-10.4 / 11.9 \mathrm{~ms}$, and the upper (mean plus 1.96 standard deviation) and lower (mean minus 1.96 standard deviation) $95 \%$ limits of agreement were $34.1 / 118.3 \mathrm{~ms}$ and $-54.9 / 94.5 \mathrm{~ms}$, respectively. Using the six-point fit, the intra/interobserver variability in $T_{1}$ was $-14.8 / 11 \mathrm{~ms}$, whereas the upper and lower $95 \%$ limits of agreement were $38.0 / 144.7 \mathrm{~ms}$ and $-67.6 / 122.7 \mathrm{~ms}$, respectively.

Figure 7 shows representative dGEMRIC $T_{1}$ maps of a 53 -year-old male patient in six rotating radial planes of the hip joint. The total scan time to acquire all six $T_{1}$ maps was 8 min. Both raw SR and PD images exhibited good image quality, and these $T_{1}$ maps depict the hip cartilage with adequate spatial resolution.

For the theoretical noise analysis, root-mean-square-error values were $27.3 \pm 1.6$ and 20.3 $\pm 1.6 \mathrm{~ms}$ for the analytic and 6-point fit $T_{1}$, respectively, compared with true $T_{1}$ ranging from 600 to $1200 \mathrm{~ms}$. Linear regression statistics were comparable between the analytic and 6-point $T_{1}$ mapping methods (see Table 2).

\section{DISCUSSION}

This study describes a new 2D $T_{1}$ mapping pulse sequence for dGEMRIC in the hip joint with a clinically acceptable scan time of 1 min 20 s per slice. Compared with a rigorous sixpoint SR acquisition ( 3 min per slice), the proposed $T_{1}$ mapping acquisition produced 
accurate results in vitro and in vivo, suggesting that the two acquisitions are quantitatively equivalent. The intra and interobserver agreements for $T_{1}$ calculations were good.

Conventional 2D $T_{1}$ mapping pulse sequences based on multipoint inversion recovery or SR with FSE readout (24-26) are clinically not feasible due to their long acquisition times. $T_{1}$ mapping pulse sequences based on gradient echo readout $(8,27)$ are more efficient than FSE based pulse sequences, but they are inherently low in SNR and sensitive to $B_{1+}$ inhomogeneities at $3 \mathrm{~T}$. Our proposed 2D pulse sequence resulted in good image quality, because we used FSE readout at $3 \mathrm{~T}$. Furthermore, it achieved uniform $T_{1}$ weighting through the use of a robust saturation pulse (20). This robust saturation pulse has been shown to effectively saturate the magnetization within the whole heart at $3 \mathrm{~T}(20)$. We anticipate $B_{1+}$ variation is lower within the hip than within the heart. Our phantom experiments showed that, compared with 3D F2T1 pulse sequence, our proposed 2D $T_{1}$ mapping pulse sequence yields higher SNR efficiency and lower sensitivity to $B_{1+}$ variations. We performed our phantom experiment assuming $B_{1+}$ variation as large as $20 \%$, based on our preliminary experience with hip imaging at $3 \mathrm{~T}$. We demonstrated that our proposed $T_{1}$ mapping pulse sequence is insensitive to up to $20 \% B_{1+}$ variation.

We validated our pulse sequence against a rigorous $T_{1}$ mapping method based on a six-point SR acquisition. A potential issue with this acquisition approach in vivo is patient motion. While we used affine transformation to perform image registration of the entire hip joint, there was small residual motion between images which could have affected $T_{1}$ calculation for some of the pixels. We anticipate the motion to be less of an issue for the two-point SR acquisition of $1 \mathrm{~min}$ and $20 \mathrm{~s}$ than the full six-point SR acquisition of $3 \mathrm{~min}$. One approach to further minimize the registration error is to perform interleaved acquisition between SR and PD. Another potential approach is to develop more accurate retrospective image registration methods. A limitation with our pulse sequence is that it may be sensitive to partial volume averaging along the through-plane direction, even with the preferred imaging in radial planes of the hip joint. Another limitation is that it may suffer minor loss in in-plane spatial resolution due to apodization in $k$-space with echo-train-length of 13 .

The mean $T_{1}$ of cartilage was on the order of $800 \mathrm{~ms}$. As such, our choice TD $=700 \mathrm{~ms}$ for the SR image acquisition represents a good balance between $T_{1}$ sensitivity and SNR, and TR $=4000 \mathrm{~ms}$ for the PD image acquisition was sufficient. For imaging tissues with different $T_{1}$, both TD for SR and TR for PD acquisitions need to be adjusted.

In conclusion, we developed a new high-resolution 2D $T_{1}$ mapping sequence, suitable for dGEMRIC in radial planes of the hip at $3 \mathrm{~T}$. We showed that the $T_{1}$ measurements are accurate, repeatable, and reproducible. The technique could be applied to measure cartilage $T_{1}$ in other joints, and it is particularly suitable for applications at $3 \mathrm{~T}$, because it is insensitive to $B_{1+}$ inhomogeneities.

\section{Acknowledgments}

Grant sponsor: American Heart Association; Grant number: 0730143N. 


\section{REFERENCES}

1. Ganz R, Parvizi J, Beck M, Leunig M, Notzli H, Siebenrock KA. Femoroacetabular impingement: a cause for osteoarthritis of the hip. Clin Orthop Relat Res. 2003; 417:112-120.

2. Wagner S, Hofstetter W, Chiquet M, Mainil-Varlet P, Stauffer E, Ganz R, Siebenrock KA. Early osteoarthritic changes of human femoral head cartilage subsequent to femoro-acetabular impingement. Osteoarthritis Cartilage. 2003; 11:508-518. [PubMed: 12814614]

3. Tanzer M, Noiseux N. Osseous abnormalities and early osteoarthritis: the role of hip impingement. Clin Orthop Relat Res. 2004; 429:170-177.

4. Kubo T, Horii M, Harada Y, Noguchi Y, Yutani Y, Ohashi H, Hachiya Y, Miyaoka H, Naruse S, Hirasawa Y. Radial-sequence magnetic resonance imaging in evaluation of acetabular labrum. $\mathbf{J}$ Orthop Sci. 1999; 4:328-332. [PubMed: 10542035]

5. Beck M, Leunig M, Parvizi J, Boutier V, Wyss D, Ganz R. Anterior femoroacetabular impingement: part II. Midterm results of surgical treatment. Clin Orthop Relat Res. 2004; 418:67-73.

6. Bashir A, Gray ML, Burstein D. Gd-DTPA2-as a measure of cartilage degradation. Magn Reson Med. 1996; 36:665-673. [PubMed: 8916016]

7. Bashir A, Gray ML, Hartke J, Burstein D. Nondestructive imaging of human cartilage glycosaminoglycan concentration by MRI. Magn Reson Med. 1999; 41:857-865. [PubMed: 10332865]

8. Kim YJ, Jaramillo D, Millis MB, Gray ML, Burstein D. Assessment of early osteoarthritis in hip dysplasia with delayed gadolinium-enhanced magnetic resonance imaging of cartilage. J Bone Joint Surg Am. 2003; 85-A:1987-1992. [PubMed: 14563809]

9. Sur S, Mamisch TC, Hughes T, Kim YJ. High resolution fast T1 mapping technique for dGEMRIC. J Magn Reson Imaging. 2009; 30:896-900. [PubMed: 19787737]

10. Mamisch TC, Dudda M, Hughes T, Burstein D, Kim YJ. Comparison of delayed gadolinium enhanced MRI of cartilage (dGEMRIC) using inversion recovery and fast T1 mapping sequences. Magn Reson Med. 2008; 60:768-773. [PubMed: 18816842]

11. Jessel RH, Zilkens C, Tiderius C, Dudda M, Mamisch TC, Kim YJ. Assessment of osteoarthritis in hips with femoroacetabular impingement using delayed gadolinium enhanced MRI of cartilage. J Magn Reson Imaging. 2009; 30:1110-1115. [PubMed: 19856439]

12. Domayer SE, Mamisch TC, Kress I, Chan J, Kim YJ. Radial dGEMRIC in developmental dysplasia of the hip and in femoroacetabular impingement: preliminary results. Osteoarthritis Cartilage. 2010; 18:1421-1428. [PubMed: 20727414]

13. Bittersohl B, Steppacher S, Haamberg T, Kim YJ, Werlen S, Beck M, Siebenrock KA, Mamisch TC. Cartilage damage in femoroacetabular impingement (FAI): preliminary results on comparison of standard diagnostic vs delayed gadolinium-enhanced magnetic resonance imaging of cartilage (dGEMRIC). Osteoarthritis Cartilage. 2009; 17:1297-1306. [PubMed: 19446663]

14. Shepherd DE, Seedhom BB. Thickness of human articular cartilage in joints of the lower limb. Ann Rheum Dis. 1999; 58:27-34. [PubMed: 10343537]

15. Stollberger, R.; Birngruber, T. In-vivo assessment of a STEAM sequence for B1-mapping. Proceedings of the 16th Annual Meeting of ISMRM; Toronto, Canada. 2008. p. 3091

16. Feiweier, T.; Heubes, P. Method and magnetic resonance tomography apparatus for spatially resolved measurement of the B1 field distribution. US Patent. 7,038,453 B2. 2006.

17. Gold GE, Han E, Stainsby J, Wright G, Brittain J, Beaulieu C. Musculoskeletal MRI at 3.0 T: relaxation times and image contrast. AJR Am J Roentgenol. 2004; 183:343-351. [PubMed: 15269023]

18. Haacke, E.; Brown, R.; Thompson, M.; Venkatesan, R. Magnetic resonance imaging. New York: Wiley; 1999. Spin density, T1 and T2 quantification methods in MR imaging; p. 637-667.

19. Griswold MA, Jakob PM, Heidemann RM, Nittka M, Jellus V, Wang J, Kiefer B, Haase A. Generalized autocalibrating partially parallel acquisitions (GRAPPA). Magn Reson Med. 2002; 47:1202-1210. [PubMed: 12111967]

20. Kim D, Oesingmann N, McGorty K. Hybrid adiabatic-rectangular pulse train for effective saturation of magnetization within the whole heart at 3 T. Magn Reson Med. 2009; 62:1368-1378. [PubMed: 19785021] 
21. Firbank MJ, Coulthard A, Harrison RM, Williams ED. A comparison of two methods for measuring the signal to noise ratio on MR images. Phys Med Biol. 1999; 44:N261-N264. [PubMed: 10616158]

22. Hansen, E.; Walster, G. Global optimizing using interval analysis: revised and expanded. New York: Marcel Dekker, Inc.; 2003.

23. Bland JM, Altman DG. Statistical methods for assessing agreement between two methods of clinical measurement. Lancet. 1986; 1:307-310. [PubMed: 2868172]

24. Crawley AP, Henkelman RM. A comparison of one-shot and recovery methods in T1 imaging. Magn Reson Med. 1988; 7:23-34. [PubMed: 3386519]

25. Haase A. Snapshot FLASH MRI. Applications to T1, T2, and chemical-shift imaging. Magn Reson Med. 1990; 13:77-89. [PubMed: 2319937]

26. Look D, Locker D. Time saving in measurement of NMR and EPR relaxation times. Rev Sci Instrum. 1970; 41:250-251.

27. Deoni SC, Rutt BK, Peters TM. Rapid combined T1 and T2 mapping using gradient recalled acquisition in the steady state. Magn Reson Med. 2003; 49:515-526. [PubMed: 12594755] 


\section{Saturation $\longleftarrow$ TD $\longrightarrow$ FSE Readout}

a

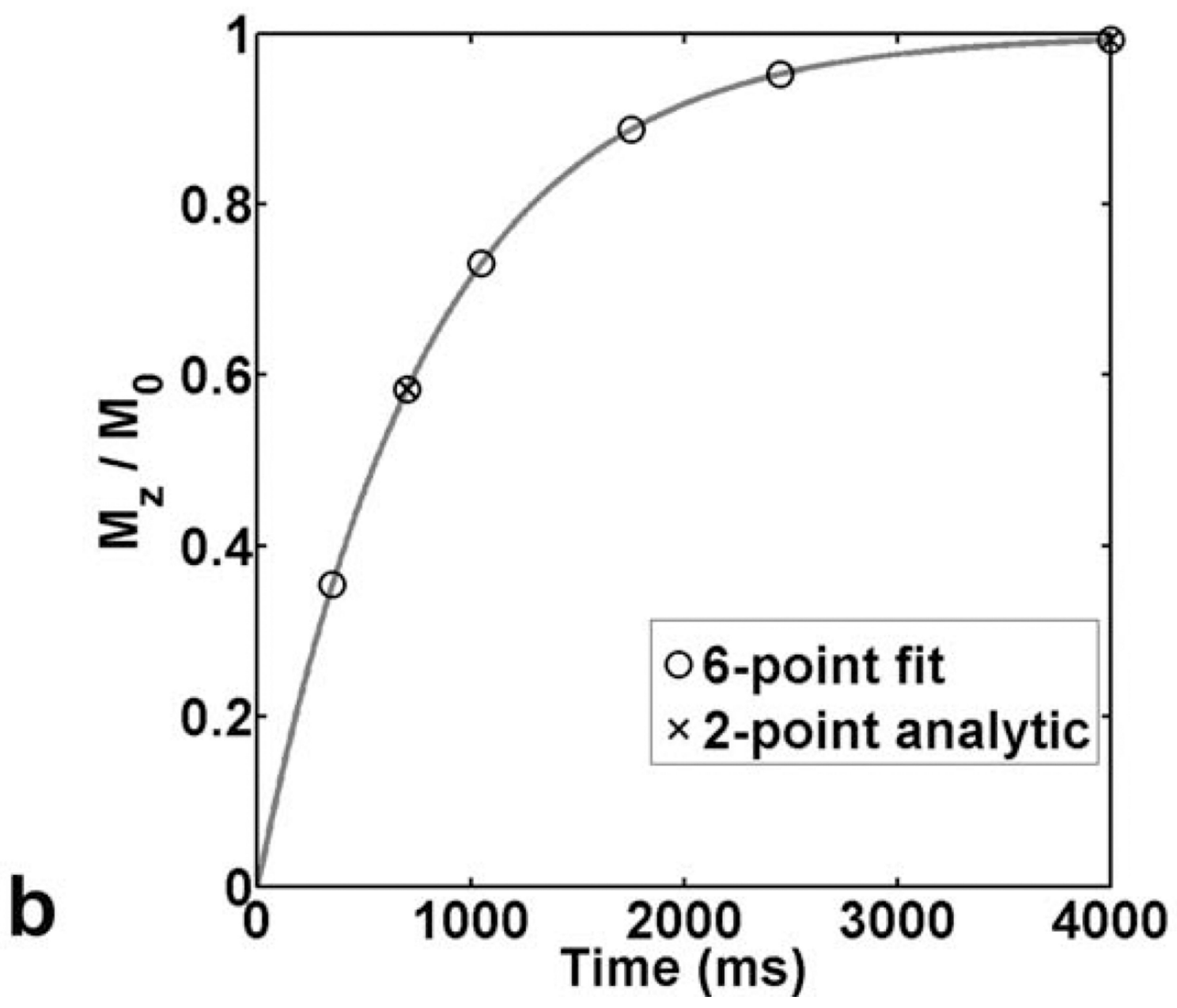

FIG. 1.

Plot of SR acquisition used in this study. The five SR acquisitions were acquired with TDs 350, 700, 1050, 1750, and $2450 \mathrm{~ms}$. The PD acquisition was acquired with TR $=4000 \mathrm{~ms}$ and without the saturation pulse. The analytical T1 measurement was made using the SR image with TD $=700 \mathrm{~ms}$ and PD image (i.e., Eq. 1). The two-parameter fit of the ideal SR equation was made using all six images. Note that all six images were acquired in series, to minimize image registration errors. 


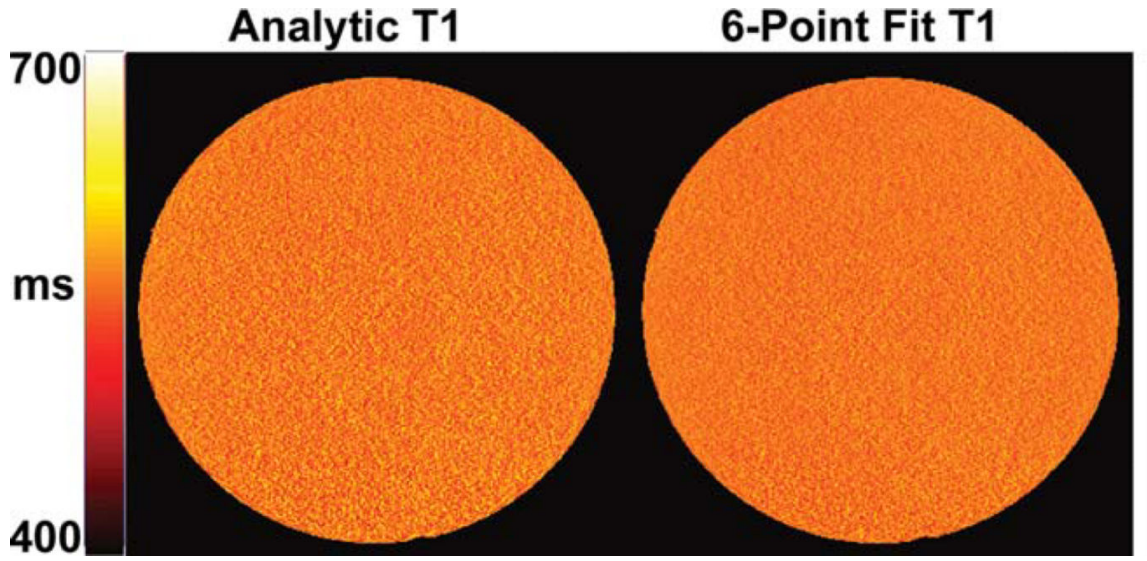

Percent Difference

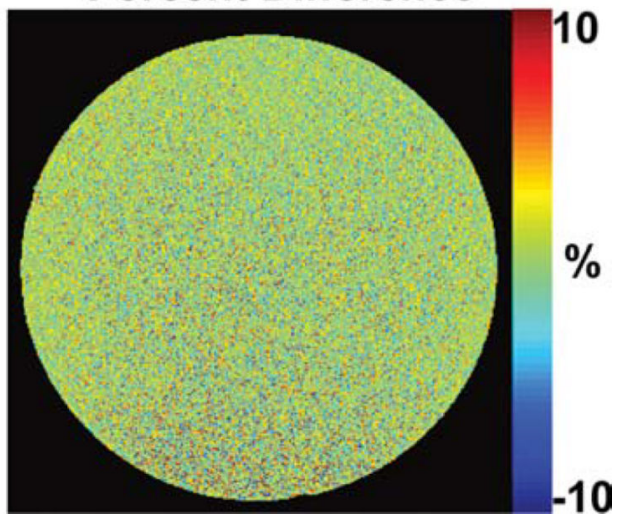

FIG. 2.

$T_{1}$ maps were calculated using the analytic and the six-point fit method for a spherical mineral oil phantom with a known $T_{1}(\sim 550 \mathrm{~ms})$. The phantom was imaged on a coronal plane without the fat suppression pulse. The difference between the two $T_{1}$ maps was calculated pixel-wise for the entire phantom. 


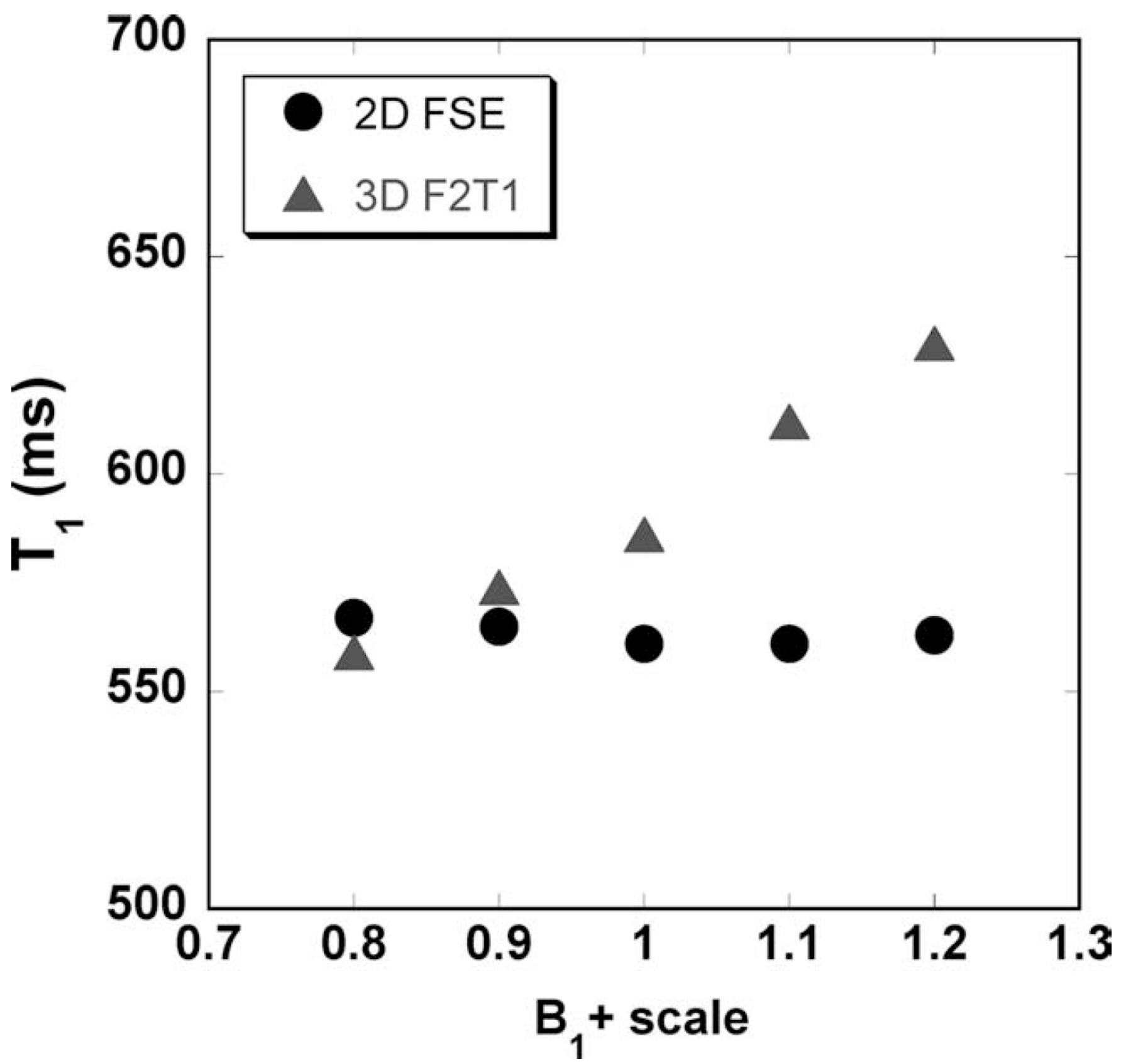

FIG. 3.

$T_{1}$ measurements as a function of $B_{1+}$ scale ranging from 0.8 to 1.2 (0.1 steps). The 3D F2T1 pulse sequence was sensitive to $B_{1+}$ scale ranging from 0.8 to 1.2 , whereas our proposed 2D $T_{1}$ mapping pulse sequence was insensitive to the same $B_{1+}$ scale range. 
$\mathrm{TD}=350 \mathrm{~ms}$

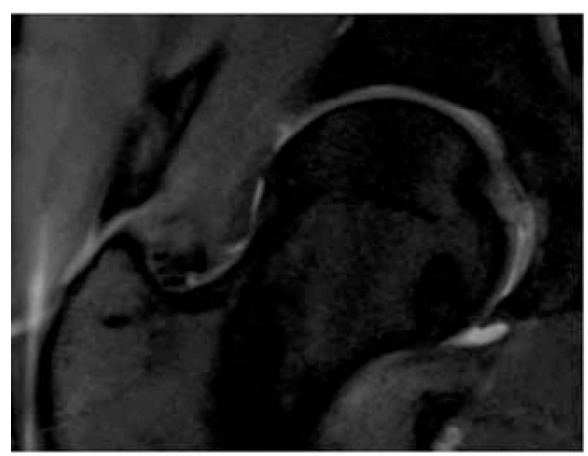

\section{$\mathrm{TD}=1750 \mathrm{~ms}$}

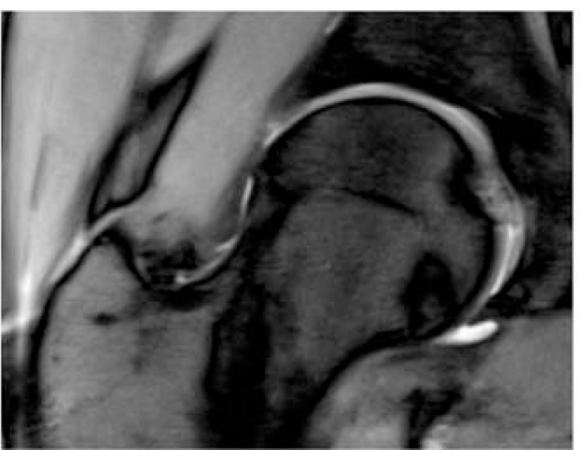

$\mathrm{TD}=700 \mathrm{~ms}$

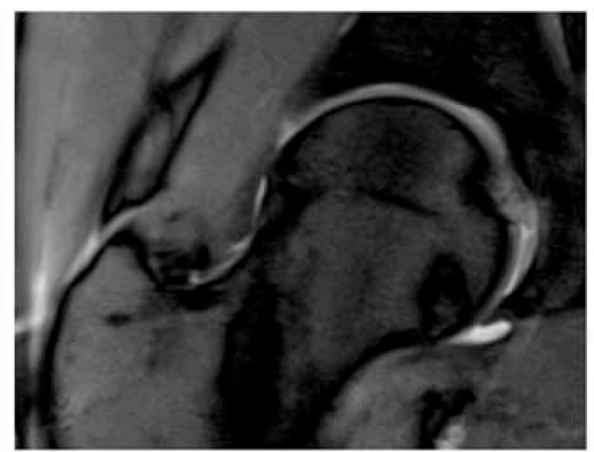

$\mathrm{TD}=2450 \mathrm{~ms}$

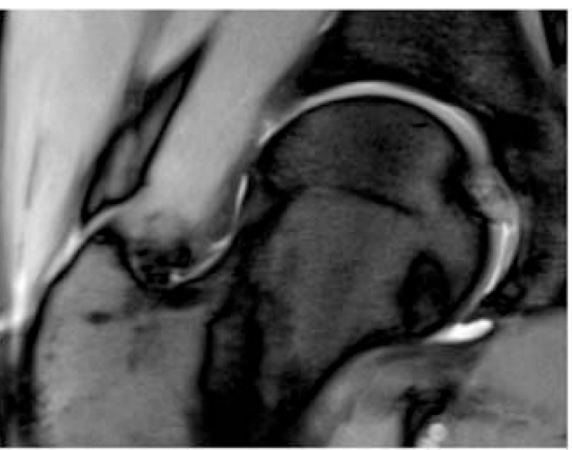

$\mathrm{TD}=1050 \mathrm{~ms}$

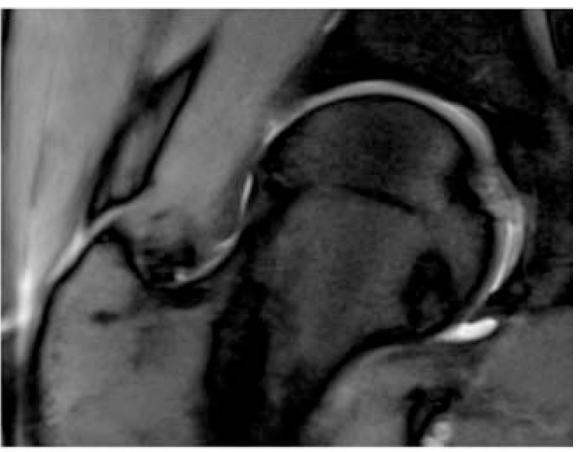

\section{$\mathrm{TD}=4000 \mathrm{~ms}$}

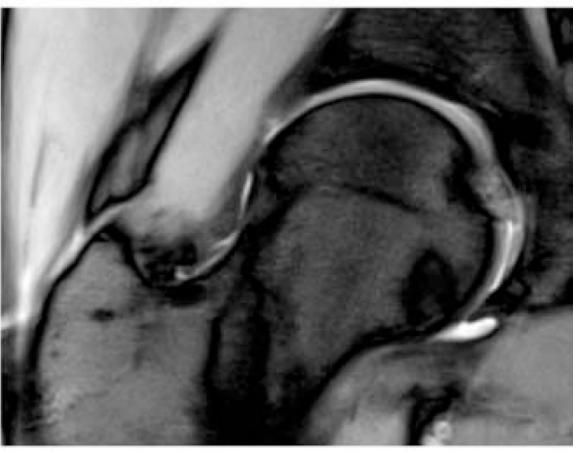

FIG. 4.

Six radial images acquired with different TD for one of the hip used in the study. $T_{1}$ was calculated rigorously by fitting the SR curve with the signals of the six images. $T_{1}$ was also calculated with the analytic formula in Eq. 1 using the second and last image. TD values were chosen assuming $T_{1}$ in the order of 700-800 $\mathrm{ms}$ in healthy hip cartilage at $3 \mathrm{~T}$, so that the image at TD $1 / 44 \mathrm{~s}$ corresponds to proton density. 
Analytic T1
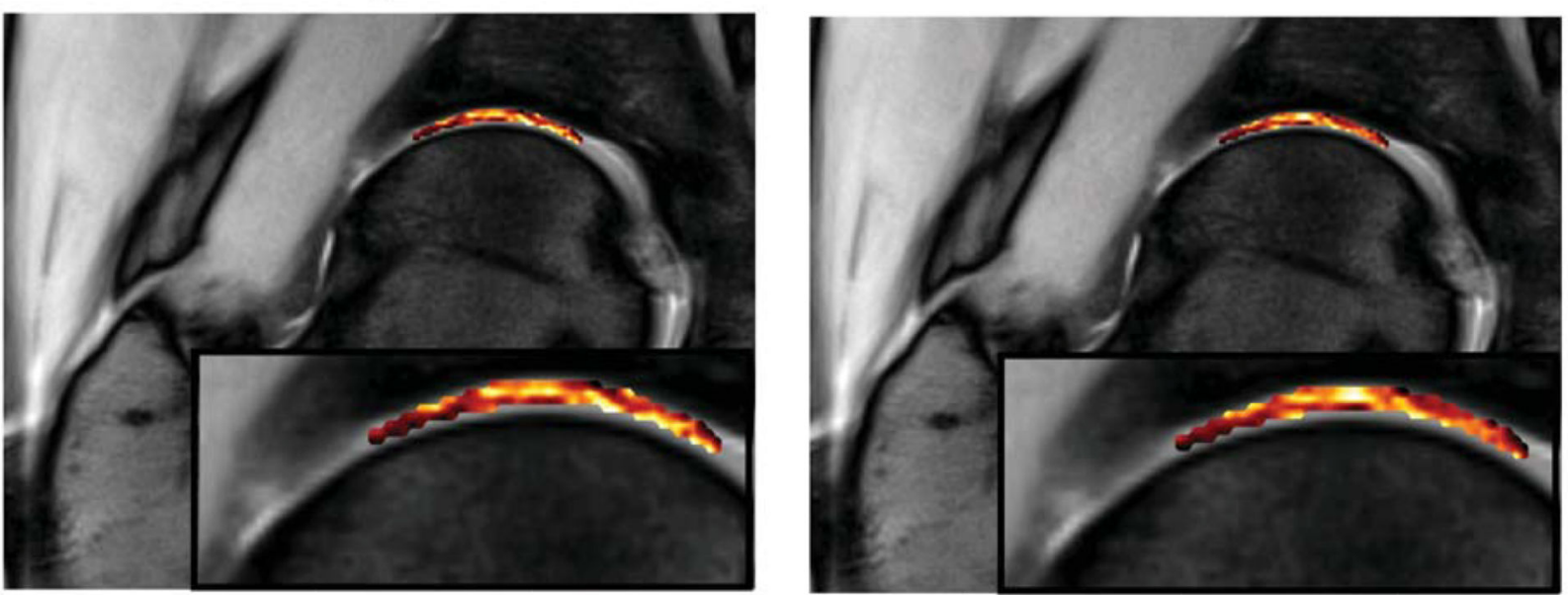

870

[ms]

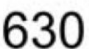

\section{Difference}
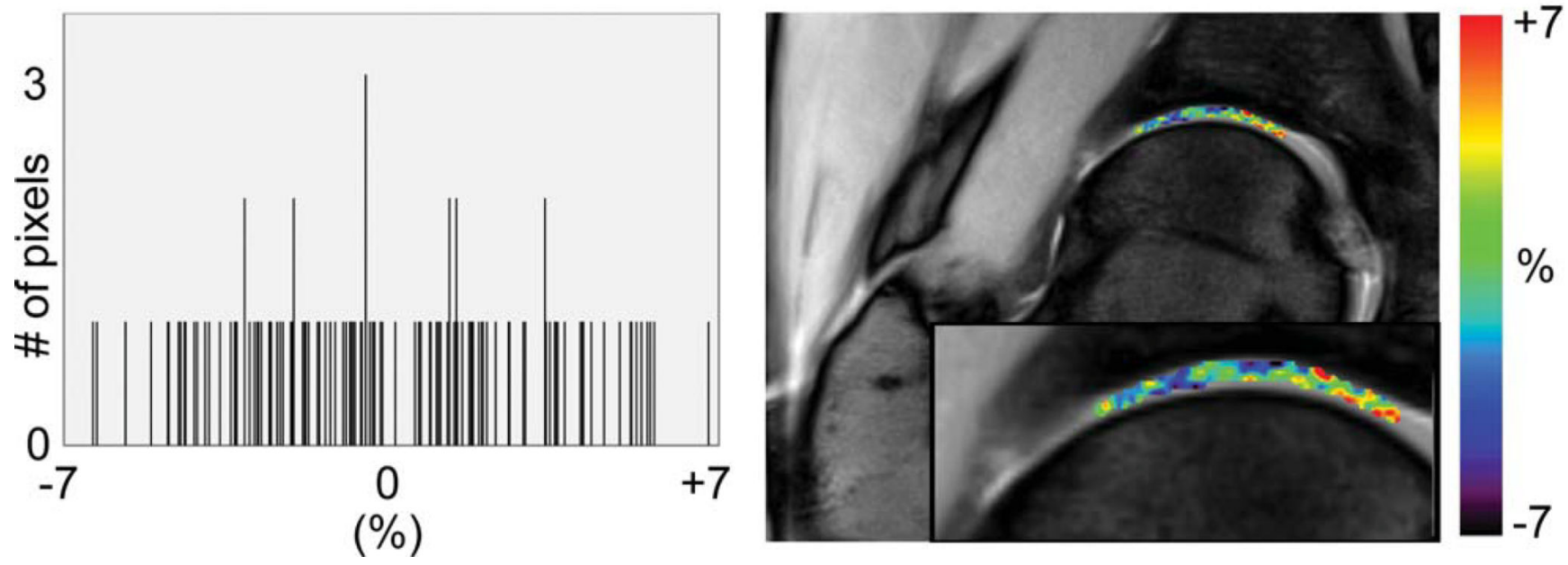

FIG. 5.

For all cases, the weight-bearing portion of hip cartilage was segmented from the lateral bony edge to the edge of the acetabular fossa. $T_{1}$ maps were calculated using the analytic and the 6-point fit method for each ROI (top row) and the percent difference between the two was computed pixel-wise (bottom row). The RMS of percent difference was $3.2 \%$ for the hip in this figure. 


\section{Observer 1, Session $1 \quad$ Observer 1, Session 2}

Observer 2
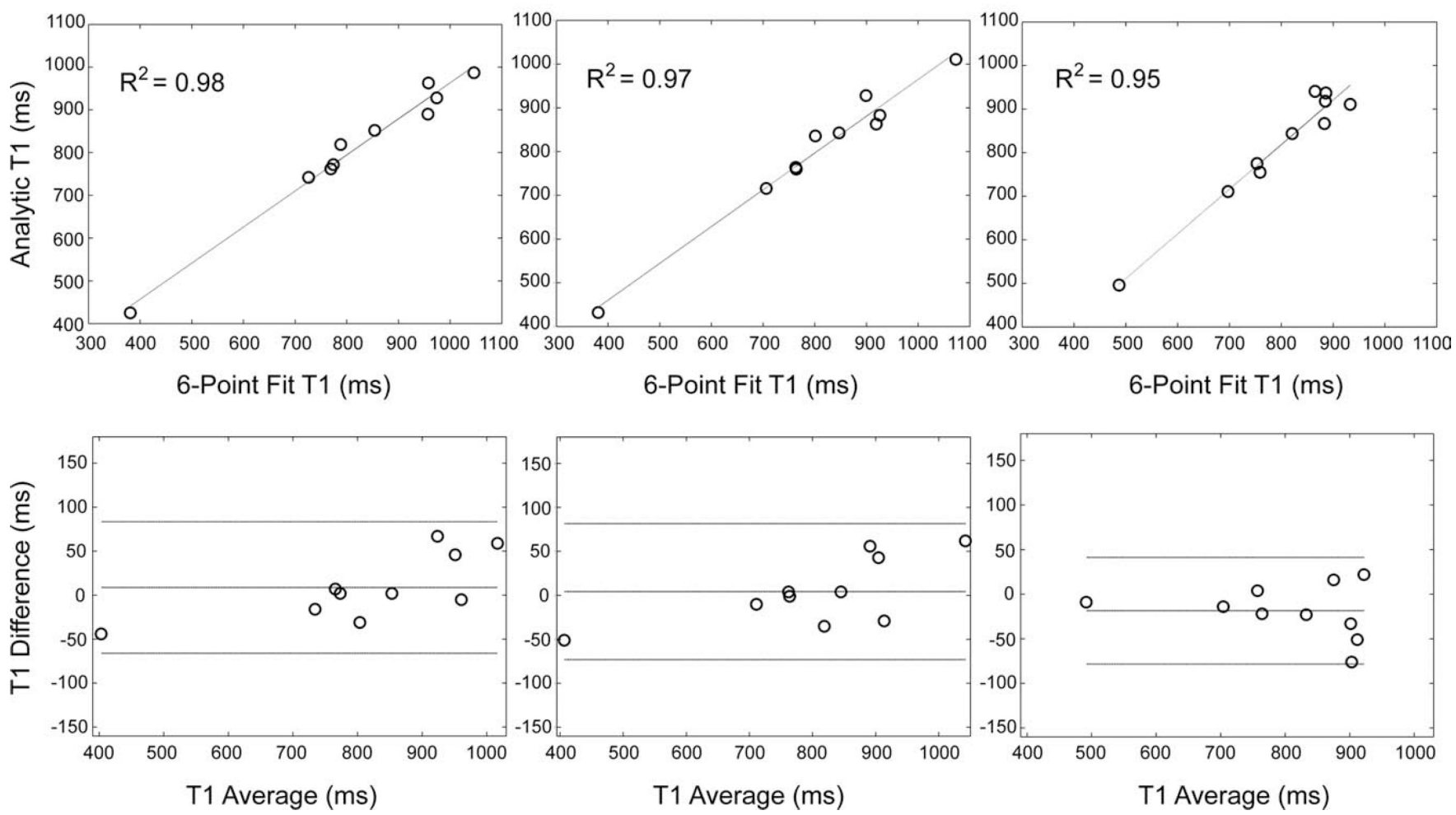

FIG. 6.

Average $T_{1}$ of the hip cartilage calculated with the analytic $T_{1}$ mapping sequence vs. rigorous 6-point fitting at $3 \mathrm{~T}$. The top row shows correlation plots and the bottom row shows Bland-Altman plots (95\% confidence intervals are displayed) of the $T_{1}$ values calculated with the two methods. The three columns correspond to the two test sessions of observer 1 and the single session of observer 2 . 


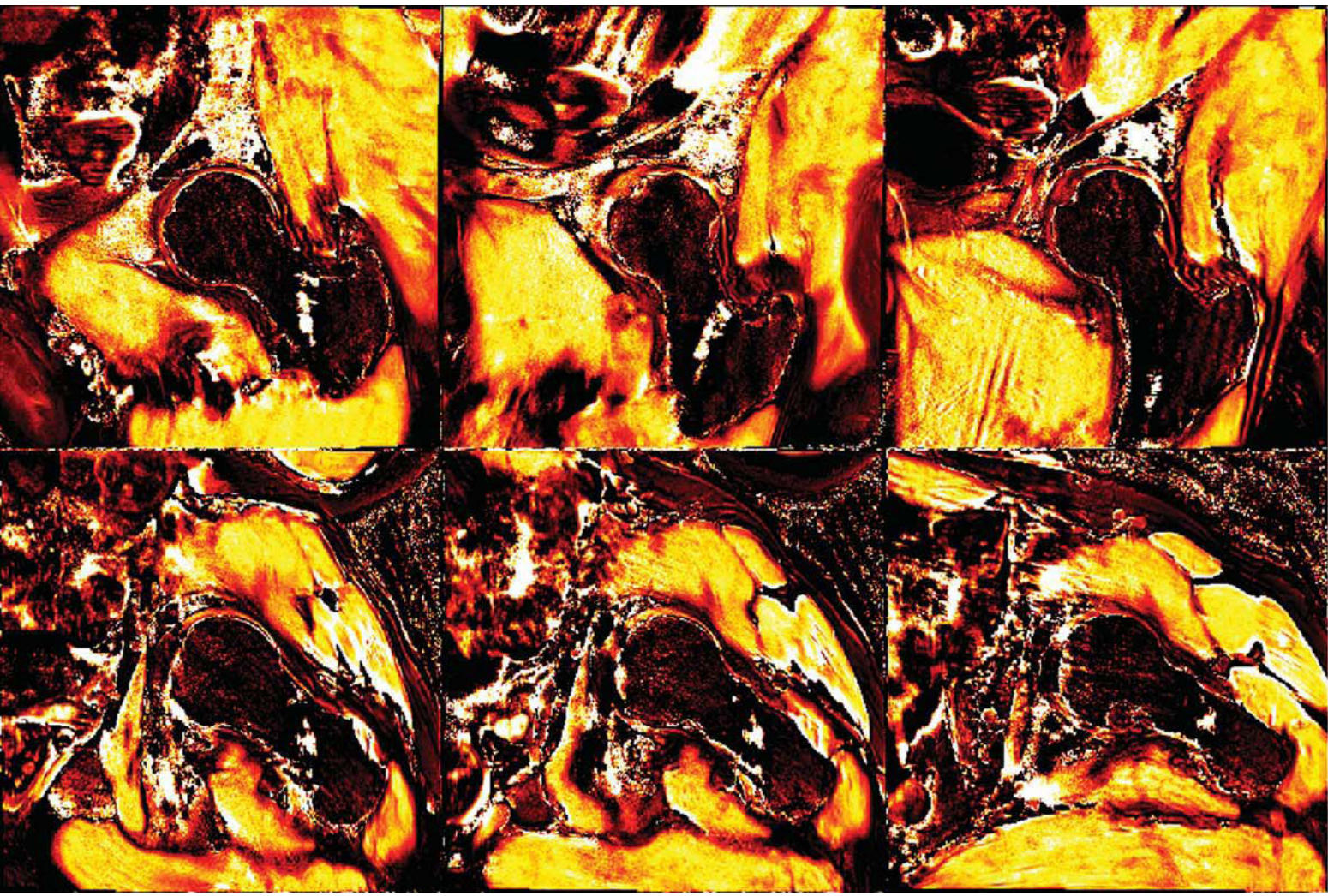

1400

FIG. 7.

Representative dGEMRIC $T_{1}$ maps a 53-year-old male patient in six rotating radial planes of the hip joint at $3 \mathrm{~T}$. These $T_{1}$ maps depict the hip cartilage with adequate spatial resolution.

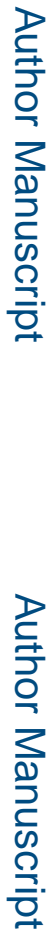




\section{Table 1}

Summary of Bland-Altman and Pearson Analysis

\begin{tabular}{|c|c|c|c|c|}
\hline Agreement type & $\begin{array}{r}\text { Difference } \\
(\mathbf{m s})\end{array}$ & $\begin{array}{r}\text { Upper } 95 \% \\
\text { limit (ms) }\end{array}$ & $\begin{array}{r}\text { Lower 95\% } \\
\text { limit (ms) }\end{array}$ & $\begin{array}{c}R^{2} \\
\text { (Pearson) }\end{array}$ \\
\hline Analytic_6-point fit (observer 1, session 1) & -8.7 & 64.5 & -81.9 & 0.98 \\
\hline Analytic—6-point fit (observer 1, session 2) & -4.3 & 71.5 & -80.1 & 0.97 \\
\hline Analytic-6-point fit (observer 2) & 18.6 & 77.2 & -40.0 & 0.95 \\
\hline \multirow{2}{*}{$\begin{array}{l}\text { Intra-observer (session } 2 \text { - session } 1 \text { : } \\
\text { analytic in gray, 6-point fit in white) }\end{array}$} & -10.4 & 34.1 & -54.9 & 0.98 \\
\hline & -14.8 & 38.0 & -67.6 & 0.98 \\
\hline \multirow{2}{*}{$\begin{array}{l}\text { Inter-observer (observer 2-observer 1: } \\
\text { analytic in gray, 6-point fit in white) }\end{array}$} & 11.9 & 118.3 & -94.5 & 0.88 \\
\hline & 11 & 144.7 & -122.7 & 0.92 \\
\hline
\end{tabular}




\section{Table 2}

Linear Regression Statistics for the Theoretical Noise Analysis, Using Eq. 1 for Reference $T_{1}$ Mapping (i.e., 6-Point SR Experiment) and Eq. 2 for Analytic $T_{1}$ Mapping, as a Function of True $T_{1}$ Ranging from 600 to $1200 \mathrm{~ms}$ (5 ms Steps)

\begin{tabular}{lcccc}
\hline Measurement pair & Slope & Bias $(\mathbf{m s})$ & $\boldsymbol{R}^{\mathbf{2}}$ & RMSE $(\mathbf{m s})$ \\
\hline Analytic $T_{1}$ vs. true $T_{1}$ & $1.01 \pm 0.01$ & $9.60 \pm 9.08$ & $0.99 \pm 0.00$ & $27.3 \pm 1.6$ \\
6-Point fit $T_{1}$ vs. true $T_{1}$ & $1.00 \pm 0.01$ & $0.35 \pm 9.26$ & $0.99 \pm 0.00$ & $20.3 \pm 1.6$ \\
\hline
\end{tabular}

Assuming $M_{0}=\mathrm{PD}$, Clinically relevant white Gaussian noise was estimated as $0.0105 M_{0}\left(=M_{0} / 95\right)$. The theoretical noise analysis was repeatedly performed 100 times using a numerical phantom with 100 pixels to mimic the typical number of pixels in the segmented hip cartilage, where identical amount of white noise was added to the numerical PD and SR images. RMSE values are $27.3 \pm 1.6$ and $20.3 \pm 1.6 \mathrm{~ms}$ for the analytic and 6-point fit $T_{1}$, respectively, compared with True $T_{1}$ ranging from 600 to $1200 \mathrm{~ms}$. 